\title{
COMPARISON BETWEEN DIFFERENT METHODS FOR DIAGNOSIS OF ACANTHAMOEBA INFECTION AND ISOLATION OF GENOTYPE T9 FROM A CONTACT LENS CASES

\author{
By
}

MOHAMMED M. ABDEL-GHAFFAR, MONA M. EL SOBKY, NANCY M. HARBA, AMANY A. RADY AND NOHA A. ABOKHALIL*

Department of Medical Parasitology, Faculty of Medicine, Menoufia University, Egypt

( ${ }^{*}$ Correspondence: drnoha_abokhalil@yahoo.com)

Abstract

Acanthamoeba genus is a medically important free living amoeba causing serious humans infections. Amoebic keratitis (AK) is a sight threatening infection of cornea caused by Acanthamoeba pathogenic genotypes, which prevalence remarkably increased in developed countries. The study compared different methods for diagnosing AK and identified Acanthamoeba genotypes by molecular examination in contact lens wearers (CLWs). Patients were 79 clinical corneal swaps (CS) and 15 samples from contact lens storage cases (CLSC). Clinical CSs were divided into four groups; GI: 20 patients suffering from chronic corneal ulcers, GII: 15 patients with traumatic ulcers, GIII: 24symptomatic CLWs and GIV: 20asymptomatic control individuals. CLSC were provided from apparently healthy asymptomatic CLWs (15). Swabs and solution samples were underwent microscopic and staining examination, cultivation on non-nutrient agar (NNA) plates and PCR molecular analysis. Sequencing and genotyping of PCR-positive samples were performed.

The results showed that Acanthamoeba parasites were detected in $3.8 \%$ of CS and $6.7 \%$ of CLSC samples. The highest significantly positive results were by culture $(3.8 \%)$ followed by Giemsa and trichrome stains $(2.5 \%)$ and lastly direct microscopy (1.3\%) of CS samples. Only one positive sample $(6.7 \%)$ was detected in CLSC by all methods, but without statistical significance. Sensitivity of PCR compared to culture was $25 \%$.

Acanthamoeba parasites in CS were from subgroup II with $12.5 \%$ detection rate in CLWs, but the positive case from CLSC was from subgroup I with $6.7 \%$ detection rate. This study confirmed different risk factors in association with AK in CLWs. Genotype determination for Acanthamoeba positive case by PCR revealed homology with Acanthamoeba genotype T9 isolate ICS20.

Key words: Acanthamoeba, Amoebic keratitis, AK, Contact Lens, CLSC, Genotype T9.

\section{Introduction}

Acanthamoeba is one of the ubiquitous free-living amoebae (FLA) of worldwide distribution (Aghajani et al, 2016). It exists as either a vegetative trophozoites or dormant cysts (Gomes et al, 2016). Under environmental stress conditions, Acanthamoeba phenotype switched from trophozoites into cysts stages (Rezaeian et al, 2008). Acanthamoeba species have been isolated from soil, air, drinking water, swimming pools, sewage, eyewash solutions, contact lenses, dialysis and dental treatment units (Cateau et al, 2014). Acanthamoeba pathogenic genotypes can cause different infections in man resulting in fatal granulomatous amoebic encephalitis (GAE) and AK which is a sight threatening infection to the cornea (Visvesvara, 2013). Acanthamoeba keratitis usually starts by pain, photo-phobia and lacrimation and progresses leading to ring ulcers, corneal opacity and corneal perforation (LorenzoMorales et al, 2015). The AK prevalence remarkably increased in developed countries, due to increased CLWs number, accurate diagnosis and disease worldwide awareness (Dart et al, 2009).

Identification of Acanthamoeba spp. depended mainly on morphological characters (Page, 1988). Molecular methods, especially PCR based on analysis of the diagnostic fragment 3 (DF3) region of 18s rRNA genes are recently used for identification. To date, 20 Acanthamoeba genotypes (T1-T20) have been established and accepted (Behera et al, 2016). Genotype T4 is the commonest and abundant pathogenic isolate from clinical cases (Khan, 2006; Maciver et al, 2013). Other genotypes; including T2, T3, T5, T6, T11 \& $\mathrm{T} 15$; were isolated and related to 
clinical manifestations (Lorenzo-Morales et al, 2011; Omaña-Molina et al, 2016).

The present study aimed to compare the molecular biology with the conventional methods of Acanthamoeba detection, also to ascertain the isolation and identification of the Acanthamoeba genotypes from AK patients at our institution.

\section{Materials and Methods}

Study population: This is a cross sectional study included 79 randomly selected individuals (45 females \& 34 males) of different age groups, 46 from Outpatient Clinic of Ophthalmology, Menoufia University Hospitals and 33 individuals from Ophthalmology Hospital in Shebin El-Koum. The study was carried out from January 2014 to April 2015. Written informed consents were obtained from all participants. They were divided into four groups; G1: 20 patients suffering from resistant corneal ulcer not responding to medical treatment for more than 2 weeks, GII: 15 patients with traumatic ulcers, GIII: 24 symptomatic CLWs and GIV: 20 asymptomatic control individuals. The study was carried out on CS. Also, 15 samples from CLSC were provided from apparently healthy asymptomatic CLWs. All participants underwent an ophthalmic examination by the ophthalmologist.

A structured questionnaire: It included demographic data, complaint and risk factors as trauma, exposure to contaminated water, history of previous keratitis and socioeconomic standard(SES) In case of CLWs, signs of over-use and incompliance to CL hygiene (including sleeping in contact lenses, inadequate cleaning of lenses with contact lens solution, \& frequency of changing contact lenses...etc.) were investigated.

Specimen collection: Corneal swabs using sterilized cotton swab were taken under complete aseptic conditions before giving any antibiotic therapy. Three swabs were obtained from each patient. One was suspended in Page's amoeba saline (PAS) for direct microscopic and staining examination, the second was cultivated on non-nutrient agar (NNA) plates and the third was put in a sterile Eppendorf tube with $200 \mu \mathrm{l}$ PBS and preserved at $-20^{\circ} \mathrm{C}$ for subsequent DNA extraction. Laboratory procedures were done at Parasitology Department laboratories, Menoufia University. Solution samples (15) from CLSC were collected in sterile tubes. Swabs from CLSC inner surfaces were taken for Biofilms examination. Each Biofilm swab was mixed well with the corresponding lens solution and left for 1-2 $\mathrm{h}$ before managing. The solution samples underwent the same methods of examination as CS samples.

Microscopic examination: Wet mount Giemsa (Ithoi et al, 2011) and Trichrome stained slides (Garcia and Bruckner, 1997) from saline suspension of swabs and CLSC solutions were microscopically examined for cysts and trophozoites by oil immersion lens.

Cultivation of specimens: Corneal swabs and CLSC solutions were cultivated according to Schuster et al. (2002) and LorenzoMorales et al. (2015) on 1.5\% non-nutrient agar (Agar No.1, Oxoid, Thermoscintific) prepared in PAS overlaid with thin layer of live Escherichia coli after cooling. Plates were incubated at $28 \pm 2^{\circ} \mathrm{C}$, and daily examined for Acanthamoeba growth up to 2 weeks by a light microscope (10x \& 40x objectives). Identification of organisms from positive culture plates was accomplished by direct examination and/or Giemsa-staining to characterize cysts morphology (shape, size, features of ectocysts \& endocysts) and trophozoites (acanthopodia \& pseudopodia). Cysts were measured by using an ocular micrometer scale and morphological characteristics into subgroups I, II, or III based on the criteria given by Pussard and Pons (1977).

Molecular analysis: DNA extraction from samples was performed by the QIAamp DNA Mini Kit (QIAGEN, Hilden, Germany) according to the manufacturer's instructions. DNA amplification was done using Acanthamoeba genus-specific primers, amplify a fragment of approximately 500bp of the ASA.S1 region of the 18s rRNA gene. Specific primer pair used in this study was 
the forward primer JDP1; 5-GGCCCAGAT CGTTTACCGTGAA and the reverse primer JDP2; 5-TCTCACAAGCTGCTAGGGGAG TCA (Schroeder et al, 2001). DNA amplification reaction was done (Booton et al, 2004); each tube contained $25 \mu 1$ total volumes composed of: $5 \mathrm{uL}$ template DNA, $1 \mu \mathrm{l}$ of each primer, 12.5 $\mu 1$ PCR Master Mix (DreamTaq Green PCR Master Mix, Thermo Fisher Scientific), and $5.5 \mu \mathrm{l}$ sterile deionized water. Amplification was done by a thermocycler (PerkinElmer Cestus, Norwalk, $\mathrm{CT})$. The process began with an initial denaturation step at $95^{\circ} \mathrm{C}$ for $7 \mathrm{~min}$, followed by 40 cycles of denaturation at $95^{\circ} \mathrm{C}$ for $1 \mathrm{~min}$, then primer annealing at $55^{\circ} \mathrm{C}$ for $1 \mathrm{~min}$, and extension at $72^{\circ} \mathrm{C}$ for $2 \mathrm{~min}$. The final extension occurred at $72^{\circ} \mathrm{C}$ for $15 \mathrm{~min}$. For negative control, distilled water was added instead of DNA and positive control DNA was kindly provided by Dr. Omnia Sobhy, Faculty of Medicine, Ain Shams University. A 100-1000 base pair (bp) ladder was used as a DNA size marker (Gene Ruler TM, Fermentas, Thermo Fisher Scientific). DNA amplified products were then electrophoresed using 3\% agarose gel stained with ethidium bromide $(0.5 \mu \mathrm{g} / \mathrm{ml})$ and then visualized under UV illumination.

Sequencing and genotyping: PCR products were purified using the QIAquick PCR purification kit (QIAGEN, Hilden, Germany) according to the manufacturer's instructions and sequenced in both directions. Sequencing of ASA.S1 region of the 18s rRNA gene was performed using approximately $20-25 \mathrm{ng}$ of PCR purified product, primers JDP1 or JDP2 and Big Dye Terminator technology. Genetic analysis was done on 3500 genetic DNA analyzer (Applied Biosystems, Thermo Fisher Scientific, USA). Following genotyping, the Basic Local Alignment Search Tool (BLAST) of the US National Center for Biotechnology Information (NCBI) was used to identify similar sequences to the present result. The sequences obtained by BLAST were aligned in MEGA 6 software program (Tamura et al, 2004) using the ClustalW method, and phylogenetic tree was constructed by neighbor joining algorithm based on evolutionary distances calculated from maximum composition likelihood method estimated with 1,000 bootstrap samplings (Tamura et al, 2013).

Statistical analysis: SPSS, version18, (SPSS Inc., Chicago, IL, USA) program was used. Data were computerized and analyzed. The $\chi 2$ and Fisher's exact tests examined the relation between qualitative variables. Values were considered significant if probability value was less than $0.05(\mathrm{P}<0.05)$ and highly significant when $\mathrm{P}$ value was 0.001 . Sensitivity, specificity, and diagnostic accuracy of different diagnostic methods were calculated in comparison to the gold standard test. Odds ratio (OR) and 95\% confidence intervals were computed to assess relation between risk factors and Acanthamoeba infection in contact lens user.

Ethical consideration: The ethical approval was obtained from the Committee of Research, Publications and Ethics of Faculty of Medicine, Menoufia University. All procedures were explained to the patients and written informed consents were obtained.

\section{Results}

Detection of Acanthamoeba infection in CS \& CLSC by different diagnostic methods: Total 94 samples were examined, 79 CS \& 15 samples from CLSC. Out of 79 CS, the highest positive cases were detected by culture in NNA media, Acanthamoeba spp. was identified in 3 clinical samples $(3.8 \%)$. Direct microscopic examination detected one positive case $(1.3 \%)$ and 2 positive cases $(2.5 \%)$ by Giemsa and trichrome stains while none positive was detected by PCR (0\%). Only one positive sample for Acanthamoeba spp. (6.7\%) out of 15 CLSC samples was detected by all examinations methods. No significant difference was recorded among different methods of examinations for CS and CLSC samples except for PCR technique ( $\mathrm{P}<0.05)$ (Tab. 1) (Fig.4). 
Table 1: Detection of Acanthamoeba infection in CS and CLSC by different diagnostic methods

\begin{tabular}{|l|c|c|c|c|c|c|c|c|}
\hline \multirow{2}{*}{\multicolumn{1}{|c|}{ Method }} & \multicolumn{3}{|c|}{ CS (79) } & \multicolumn{3}{c|}{ CLSC (15) } & \multicolumn{2}{c|}{ Analysis } \\
\cline { 2 - 9 } & Positive & Negative & Positive \% & Positive & Negative & Positive \% & $\chi 2$ & P \\
\hline Direct microscopy & 1 & 78 & $1.3 \%$ & 1 & 14 & $6.7 \%$ & 1.766 & 0.184 \\
\hline Giemsa stain & 2 & 77 & $2.5 \%$ & 1 & 14 & $6.7 \%$ & 0.698 & 0.404 \\
\hline Trichrome stain & 2 & 77 & $2.5 \%$ & 1 & 14 & $6.7 \%$ & 0.698 & 0.404 \\
\hline Culture & 3 & 76 & $3.8 \%$ & 1 & 14 & $6.7 \%$ & 0.255 & 0.614 \\
\hline PCR & 0 & 79 & $0.0 \%$ & 1 & 14 & $6.7 \%$ & 5.323 & 0.021 \\
\hline
\end{tabular}

Positive samples were detected on patients using contact lenses (GIII); rate was $12.5 \%$ by culture. Staining methods detected 2 pos-

itive cases $(8.3 \%)$, but without significance difference between examination methods Table 2: Distribution of demographic features of the examined CL cases in relation to Acanthamoeba infection.

\begin{tabular}{|c|c|c|c|c|c|c|c|c|c|c|c|}
\hline \multirow{3}{*}{\multicolumn{2}{|c|}{ Risk factor }} & \multicolumn{4}{|c|}{ Acanthamoeba } & \multirow{2}{*}{\multicolumn{2}{|c|}{ Total }} & \multirow{3}{*}{$\begin{array}{l}\text { Odds } \\
\text { ratio }\end{array}$} & \multirow{2}{*}{\multicolumn{2}{|c|}{$\begin{array}{c}95 \% \text { confidence } \\
\text { interval }\end{array}$}} & \multirow{3}{*}{$\begin{array}{c}\mathrm{P} \\
\text { value }\end{array}$} \\
\hline & & \multicolumn{2}{|c|}{ +ve $(3 / 24)$} & \multicolumn{2}{|c|}{-ve $(21 / 24)$} & & & & & & \\
\hline & & No. & $\%$ & No & $\%$ & No & $\%$ & & Upper & Lower & \\
\hline \multirow{2}{*}{ Sex } & $\mathrm{M}$ & 1 & $33.3 \%$ & 5 & $22.7 \%$ & 6 & $24.0 \%$ & \multirow{2}{*}{1.700} & \multirow{2}{*}{0.126} & \multirow{2}{*}{22.873} & \multirow{2}{*}{1.00} \\
\hline & $\mathrm{F}$ & 2 & $66.7 \%$ & 17 & $77.3 \%$ & 19 & $76.0 \%$ & & & & \\
\hline \multirow{2}{*}{ Age group } & $15-<30$ & 0 & $0.0 \%$ & 17 & $81.0 \%$ & 17 & $70.8 \%$ & \multirow{2}{*}{0.069} & \multirow{2}{*}{0.006} & \multirow{2}{*}{0.769} & \multirow{2}{*}{0.026} \\
\hline & $>30-45$ & 3 & $100.0 \%$ & 4 & $19.0 \%$ & 7 & $29.2 \%$ & & & & \\
\hline \multirow{2}{*}{ SES } & High & 1 & $33.3 \%$ & 3 & $14.3 \%$ & 4 & $16.7 \%$ & \multirow{2}{*}{3.000} & \multirow{2}{*}{0.203} & \multirow{2}{*}{44.359} & \multirow{2}{*}{0.44} \\
\hline & Moderate & 2 & $66.7 \%$ & 18 & $85.7 \%$ & 20 & $83.3 \%$ & & & & \\
\hline \multirow{2}{*}{ Residence } & Rural & 0 & $0 \%$ & 2 & $9.5 \%$ & 2 & $8.3 \%$ & \multirow{2}{*}{33.0} & \multirow{2}{*}{2.248} & \multirow{2}{*}{484.45} & \multirow{2}{*}{0.011} \\
\hline & urban & 3 & $100 \%$ & 19 & $90.5 \%$ & 22 & $91.7 \%$ & & & & \\
\hline
\end{tabular}

Culture was considered as a gold standard test. The highest values were of Giemsa and trichrome stain, they showed one falsenegative sample that was positive by culture yielding $75 \%$ sensitivity, $100 \%$ specificity and $99 \%$ accuracy followed by microscopy, which showed 2 false-negative samples with $50 \%$ sensitivity, $100 \%$ specificity and $98 \%$ accuracy. Lowest results were by PCR method which gave 3 false-negative samples with $25 \%$ sensitivity, $100 \%$ specificity and 97\% accuracy.

Direct microscopy and stained smears of positive samples showed Acanthamoeba trophozoites characterized by its' irregular shape, centrally placed single nucleus, large, dense nucleolus, many cytoplasmic contractile vacuoles and fine, tapering, and thornlike acanthopodia arising from the body surface. Cysts showed wrinkled or smooth ectocysts and endocysts that varied in shape, being stellate or spherical according to species. Cysts had one nucleus with central dense nucleolus (Figs.2 \& 3).

Cysts of Acanthamoeba subgroup I had rounded smooth ectocysts that were clearly separated from the endocysts. Ectocyst and endocyst were joined by radiations forming star-shaped structure with a mean diameter of more than $20 \mu \mathrm{m}$. Cysts were subgroup II had ectocysts and endocysts; either close together or widely separated. Ectocysts were wrinkled or smooth and endocysts were polygonal or round with a mean diameter of less than $18 \mu \mathrm{m}$. Most of Acanthamoeba were of subgroup II ( 3 positive clinical cases from symptomatic CLWs) and the positive isolate from CLSC was of subgroup I.

Demographic criteria in CLWs regarding Acanthamoeba infection: Acanthamoeba was significantly detected among ages $>30$ 45with $(\mathrm{P}=0.026)$. Infection was detected in female more than males. Two out of 3 positive cases $(66.7 \%)$ were females. Also, 2 out of $3(66.7 \%)$ were detected in moderate SES. Sex and the SES had no significant relation with Acanthamoeba infection (P) 0.05). All positive 3 cases for Acanthamoe$b a$ infection were detected among urban patients $(\mathrm{P}=0.011)$ (Tab.2). 
Table3: Distribution of CL hygiene and water exposure risk factors in relation to Acanthamoeba infection in symptomatic CLWs $(\mathrm{N}=24)$.

\begin{tabular}{|c|c|c|c|c|c|c|c|c|c|}
\hline \multirow{2}{*}{\multicolumn{2}{|c|}{ Risk factor }} & \multicolumn{4}{|c|}{ Acanthamoeba } & \multirow{5}{*}{$\begin{array}{c}\text { Odds } \\
\text { ratio }\end{array}$} & \multirow{2}{*}{\multicolumn{2}{|c|}{ Confidence interval $95 \%$}} & \multirow{5}{*}{$\begin{array}{c}\mathrm{P} \\
0.015\end{array}$} \\
\hline & & \multicolumn{2}{|c|}{ +ve (3) } & \multicolumn{2}{|c|}{-ve (21) } & & & & \\
\hline & & No. & $\%$ & No. & $\%$ & & lower & upper & \\
\hline \multirow{2}{*}{$\begin{array}{c}\text { Hygiene } \\
\text { awareness }\end{array}$} & good & 0 & $0 \%$ & 18 & $85.7 \%$ & & & & \\
\hline & poor & 3 & $100 \%$ & 3 & $14.3 \%$ & & 0.005 & 0.605 & \\
\hline \multirow{2}{*}{ Hand washing } & $+\mathrm{ve}$ & 1 & $33.3 \%$ & 21 & $100 \%$ & \multirow{2}{*}{0.030} & \multirow{2}{*}{0.002} & \multirow{2}{*}{0.445} & \multirow{2}{*}{0.011} \\
\hline & -ve & 2 & $66.7 \%$ & 0 & $0 \%$ & & & & \\
\hline \multirow{2}{*}{$\begin{array}{l}\text { Regular CLSC } \\
\text { cleaning }\end{array}$} & $+\mathrm{ve}$ & 1 & $33.3 \%$ & 20 & $95.2 \%$ & \multirow{2}{*}{0.025} & \multirow{2}{*}{0.001} & \multirow{2}{*}{0.572} & \multirow{2}{*}{0.032} \\
\hline & -ve & 2 & $66.7 \%$ & 1 & $4.8 \%$ & & & & \\
\hline \multirow{2}{*}{$\begin{array}{c}\text { Rinsing CL } \\
\text { with tap water }\end{array}$} & $+\mathrm{ve}$ & 2 & $66.7 \%$ & 1 & $4.8 \%$ & \multirow{2}{*}{40.000} & \multirow{2}{*}{1.749} & \multirow{2}{*}{914.787} & \multirow{2}{*}{0.032} \\
\hline & -ve & 1 & $33.3 \%$ & 20 & $95.2 \%$ & & & & \\
\hline \multirow{2}{*}{$\begin{array}{l}\text { Rinsing CLSC } \\
\text { with tap water }\end{array}$} & $+\mathrm{ve}$ & 3 & $100 \%$ & 2 & $9.5 \%$ & \multirow{2}{*}{26.667} & \multirow{2}{*}{2.178} & \multirow{2}{*}{326.453} & \multirow{2}{*}{0.008} \\
\hline & -ve & 0 & $0 \%$ & 19 & $90.5 \%$ & & & & \\
\hline \multirow{2}{*}{$\begin{array}{l}\text { Multipurpose } \\
\text { solution }\end{array}$} & $+\mathrm{ve}$ & 3 & $100 \%$ & 2 & $9.5 \%$ & \multirow{2}{*}{26.667} & \multirow{2}{*}{2.178} & \multirow{2}{*}{326.453} & \multirow{2}{*}{0.008} \\
\hline & -ve & 0 & $0 \%$ & 19 & $90.5 \%$ & & & & \\
\hline \multirow{2}{*}{$\begin{array}{l}\text { Special solu- } \\
\text { tion }\end{array}$} & $+\mathrm{ve}$ & 0 & $0 \%$ & 16 & $76.2 \%$ & \multirow{2}{*}{0.088} & 0008 & 0.054 & 0.041 \\
\hline & -ve & 3 & $100 \%$ & 5 & $23.8 \%$ & & 08 & 0. & 041 \\
\hline Showering + & $+\mathrm{ve}$ & 2 & $66.7 \%$ & 1 & $4.8 \%$ & 4000 & 1749 & 914787 & 0032 \\
\hline CL wear & -ve & 1 & $33.3 \%$ & 20 & $95.2 \%$ & 40.00 & 1.149 & 914.101 & 0.052 \\
\hline
\end{tabular}

Distribution of risk factors among CLWs to infection: There were significant associations between poor awareness, negligence of hand washing before handling CL, irregular cleaning of CL and Acanthamoeba infection ( $\mathrm{P}=0.015,0.011 \& 0.032$ respectively). Water exposure risk factors in CL wearers

Table 4: Distribution of other CL-related risk factors in relation to Acanthamoeba in symptomatic CLWs

\begin{tabular}{|c|c|c|c|c|c|c|c|c|c|}
\hline \multirow{3}{*}{\multicolumn{2}{|c|}{ Risk factor }} & \multicolumn{4}{|c|}{ Acanthamoeba } & \multirow{3}{*}{$\begin{array}{l}\text { Odds } \\
\text { ratio }\end{array}$} & \multirow{2}{*}{\multicolumn{2}{|c|}{$\begin{array}{c}\text { Confidence inter- } \\
\text { val } 95 \% \\
\end{array}$}} & \multirow{3}{*}{$\mathrm{P}$} \\
\hline & & \multicolumn{2}{|c|}{ +ve (3) } & \multicolumn{2}{|c|}{-ve (21) } & & & & \\
\hline & & No. & $\%$ & No. & $\%$ & & lower & upper & \\
\hline \multirow{2}{*}{$\begin{array}{l}\text { Sleeping +CL } \\
\text { wear }\end{array}$} & $+\mathrm{ve}$ & 2 & $66.7 \%$ & 5 & $23.8 \%$ & \multirow{2}{*}{6.400} & \multirow{2}{*}{0.474} & \multirow{2}{*}{86.343} & \multirow{2}{*}{0.194} \\
\hline & $-\mathrm{ve}$ & 1 & $33.3 \%$ & 16 & $76.2 \%$ & & & & \\
\hline \multirow{2}{*}{$\begin{array}{l}\text { Corneal trau- } \\
\text { ma }\end{array}$} & $+\mathrm{ve}$ & 3 & $100 \%$ & 4 & $19.0 \%$ & \multirow{2}{*}{14.400} & \multirow{2}{*}{1.300} & \multirow{2}{*}{159.513} & \multirow{2}{*}{0.026} \\
\hline & -ve & 0 & $0 \%$ & 17 & $81.0 \%$ & & & & \\
\hline \multirow{2}{*}{$\begin{array}{c}\text { Purpose of CL } \\
\text { wear }\end{array}$} & Optical & 2 & $66.7 \%$ & 17 & $81.0 \%$ & \multirow{2}{*}{0.471} & \multirow{2}{*}{0.034} & \multirow{2}{*}{6.568} & \multirow{2}{*}{0.521} \\
\hline & Cosmetic & 1 & $33.3 \%$ & 4 & $19.0 \%$ & & & & \\
\hline \multirow{2}{*}{$\begin{array}{l}\text { Frequency of } \\
\text { application }\end{array}$} & Daily & 2 & $66.7 \%$ & 15 & $71.4 \%$ & \multirow{2}{*}{0.800} & \multirow{2}{*}{0.061} & \multirow{2}{*}{10.562} & \multirow{2}{*}{1.000} \\
\hline & Occasionally & 1 & $33.3 \%$ & 6 & $28.6 \%$ & & & & \\
\hline \multirow{2}{*}{$\begin{array}{l}\text { Use of expired } \\
\text { CLs }\end{array}$} & $+\mathrm{ve}$ & 1 & $33.3 \%$ & 6 & $28.6 \%$ & \multirow{2}{*}{1.250} & \multirow{2}{*}{0.095} & \multirow{2}{*}{16.503} & \multirow{2}{*}{1.000} \\
\hline & -ve & 2 & $66.7 \%$ & 15 & $71.4 \%$ & & & & \\
\hline \multirow{2}{*}{ Dust exposure } & $+\mathrm{ve}$ & 3 & $100 \%$ & 5 & $23.8 \%$ & \multirow{2}{*}{11.333} & \multirow{2}{*}{1.048} & \multirow{2}{*}{122.549} & 0.041 \\
\hline & -ve & 0 & $0 \%$ & 16 & $76.2 \%$ & & & & 0.041 \\
\hline Topical ster- & $+\mathrm{ve}$ & 2 & $66.7 \%$ & 0 & $0 \%$ & & & & \\
\hline oid use & -ve & 1 & $33.3 \%$ & 21 & $100 \%$ & 33.000 & 2.248 & 484.447 & 0.011 \\
\hline
\end{tabular}

Acanthamoeba infection was significantly detected in cases with dust exposure history ( $\mathrm{P}=0.04)$, daily using $\mathrm{CL}$ than occasional ones and sleeping wearing CL (66.7\%), but without significant association $(\mathrm{P}>0.05)$. Other risk factors as the purpose of CL wear and the use of expired CL, without signifi- showed significant association between showering while wearing CL, rinsing CL or their cases with tap water, using multipurpose solution for cleaning CL, wothout use special solution $\&$ positive cases $(\mathrm{P}=0.032$, $0.008 \& 0.041$ respectively) (Tab.3). 
eba spp. in the National Center for Biotechnology (NCBI) showed homology with genotype T9 isolate ICS20. The present sequence generated was submitted to the Genbank database (accession number KR 270798) and designated as strain NA-2015.

Phylogenetic tree reconstructions using the neighbour-joining method \& MEGA6 software program placed the present Acanthamoeba spp. within genotype 9 with $71 \%$ similarity to Acanthamoeba genotype T9 isolate ICS20 and 58\% similarity with $A$. astronyxis isolate: IK-HD191 (Fig. 5).

\section{Discussion}

Acanthamoeba keratitis is an ulcerative disease of the cornea which can cause severe ocular damage, ending in complete loss of vision (Lorenzo-Morales et al, 2015).

In the present study, the Acanthamoeba was detected in $3.8 \%$ of corneal swabs and $6.7 \%$ of CLSC samples. In Egypt, the prevalence of AK was $5.26 \% \& 27.37 \%$ in corneal swabs and scraping respectively (AboulMagd et al, 2016). Also, Acanthamoeba was identified in 32/260 (12.3\%) of cases with infectious keratitis (Taher et al, 2018). The low detection rate in the current work might be attributed to corneal swabbing. These results agreed with Anisah et al. (2005) who reported that swabbing was an insensitive technique for isolation and detection of amoeba. Also, Vemuganti et al. (2000) reported that the trophozoites were in the anterior stroma, but the cysts in the deeper one.

In the present study, the highest significant positivity was by culture (3.8\%) followed by Giemsa and trichrome stains $(2.5 \%)$, microscopy (1.3\%) and lastly PCR (0\%) of CS samples. Only one positive case $(6.7 \%)$ was detected in CLSC by all methods, but without significance. Wanachiwanawin et al. (2012) reported positive rate of $15.3 \%$ for direct microscopy and $46.1 \%$ for culture. Niyyati et al. (2009) reported that corneal scrapes examination from AK patients was negative and culture was positive in $14.3 \%$. The obstacles of Acanthamoeba detection by direct smear was due to small corneal sam- ples with few parasite and required technical expertise (Qvarnstrom et al, 2006). Also, the antibiotics pre-treated patients have a very rare parasite (Lorenzo-Morales et al, 2015).

In the present study, culture was used as a gold standard test to detect Acanthamoeba. Direct smear showed 2 false-negative samples with sensitivity $50 \%$ and Giemsa stain showed one false-negative sample with $75 \%$ sensitivity. These results agreed with Boggild et al. (2009) who found that direct smear had the poorest diagnostic sensitivity (33-55\%) and Giemsa-stained smear showed $55 \%$ sensitivity, Giemsa stain differentiated nuclear and cytoplasm, without staining cysts' outer wall (Behera and Satpathy, 2016).

In the present study, trichrome stain revealed Acanthamoeba in 3/94 specimens with $75 \%$ sensitivity and $100 \%$ specificity. El-Sayed and Hikal (2015) reported that Modified trichrome was the most consistent stain for Acanthamoeba cysts and superior to Giemsa stain with high rank $(56 \%)$.

Molecular diagnosis improved AK diagnosis by amplifying Acanthamoeba DNA and detected scanty organisms in clinical cases (Laummaunwai et al, 2012). In the present study, genus-specific primers pair; JDP1 (forward) and JDP2 (reverse) were used for PCR amplification of Acanthamoe$b a$-specific nuclear small subunit ribosomal RNA; 18S rRNA gene segment or the Acanthamoeba specific amplimer-S1 (ASA-S1), which were well accepted (Gatti et al, 2010; El-Sayed et al, 2014; Tawfeek et al, 2016).

In the current study, one positive Acanthamoeba case $(6.7 \%)$ out of 15 CLSC samples was detected by PCR and all positive three corneal samples detected by culture were PCR-negative. PCR sensitivity (using JDP primers) compared to culture was $25 \%$. But, Wanachiwanawin et al. (2012) reported positivity rate of $92.3 \%$ for conventional PCR, \& $100 \%$ for real-time PCR. AboulMagd et al. (2016) reported that the highest significantly positive cases were obtained by PCR in both swabbed (5.26\%) and scraped (27.37\%) samples. Taher et al. (2018) repor- 
ted PCR positivity of $12.3 \%$.

Regarding the same primer pair, low sensitivity was obtained by Boggild et al. (2009), assumed that it might be due to the PCR inhibitor in the corneal tissue or low volume of corneal sample. Schroeder et al. (2001) attributed the low PCR sensitivity to presence of mature resistant cysts in positive sample, whereas samples with trophozoites or immature cysts were PCR positive. Likewise, Goldschmidt et al. (2008) found that the PCR-false-negative results might be due to the high resistance of Acanthamoeba cysts to reagents exposed DNA or insufficient DNA material from corneal samples. Application of local anesthesia before taking corneal samples inhibited Taq polymerase or act as PCR inhibitors (Laummaunwai et al, 2012; El-Sayed et al, 2014).

The current results showed that all Acanthamoeba infected cases were detected among CLW and CLSC, with the detection rate of $12.5 \%$ \& $6.7 \%$ respectively. In Egypt, Acanthamoeba infection was detected in $81 \%$ of CLW (El-Sayed et al, 2014). This association between AK \& CLWs was proven once upon a time by others (Ibrahim et al, 2009; Gupta and Aher 2009). Wanachiwanawin et al. (2012) diagnosed AK in $62.5 \%$ of CLWs and in $37.5 \%$ of non-contact lens wearers (NCLW). Ghamilouie et al. (2014) reported that $5.6 \%$ of keratitis patients were Acanthamoeba positive in all the contact lens wearers. Also Aboul-Magd et al. (2016) reported that Acanthamoeba infection was higher in CLWs $(34.48 \%)$ than NCLW (16.21\%) but without significance. This association may be due to trauma in the corneal epithelium during manipulation of contact lens and transmission of Acanthamoeba trophozoites to the eye (Ibrahim et al, 2009). Also, chronic hypoxic stress on corneal epithelium by continuous use of CL led to edema and significant thinning of corneal epithelium (Liesegang, 2002).

In the present study, the Acanthamoeba parasites among CLWs were from group II with detection rate $12.5 \%$ and the only one positive identified case from CLSC was from group I with detection rate of $6.7 \%$. Casero et al. (2017) reported that Acanthamoeba isolates from CL demonstrated phenotypic differentiation, where $82 \%$ of them were group II \& $18 \%$ group III. Besides, Walochnik et al. (2015) reported that group II was the predominant pathogenic clinical isolates. Buchele et al. (2018) recorded that Acanthamoeba isolate identified by cyst morphology belonged to group II.

This study called attention to the risk factors associated with AK among CLWs. Regarding demographic criteria, Acanthamoe$b a$ infection was significantly detected in cases their age group >30-45 including all positive cases, $(\mathrm{P}=0.026)$. But, Taher et al. (2018) reported that Acanthamoeba parasite was significant in age group $\geq 21$ to 25 years.

In the present study, Acanthamoeba was detected in females more than males, 2 out of 3 positive cases $(66.7 \%)$ were females. This agreed with Walochnik et al. (2015) and Taher et al. (2018). On the contrary, Ibrahim et al. (2009) revealed that the females incidence were less than males. The association of Acanthamoeba infections among females may be attributed to usage of contact lens for cosmetic purpose in youth and refusal of wearing glasses (Mahittikorn et al, 2017). Also, females usually use cosmetics as eye mascara, which could coat the CLs surfaces, allowing bacteria and/or Acanthamoeba to adhere (Srinivasan et al, 2015).

In this study, there was significant association between different risk factors related to CL hygiene and water exposure and positive cases for Acanthamoeba parasite. Lack of awareness about CL hygiene was significantly associated with AK in $34.4 \%$ of cases (Taher et al, 2018). This might result from moderate to low SES.

The CDC sent a strong message about the risk of exposure to water sources on development of AK (Legarreta et al, 2013). Also, Evyapan et al. (2015) pointed out swimming and showering while wearing CL and lack of hand washing were an important risk fac- 
tor for acquiring AK due to the forward regression analysis. Gomes et al. (2016) reported that high detection rate of Acanthamoeba in CLWs not washing hands before handling, or showering while wearing CL and in patients not cleaning the CL cases. Carnt et al. (2018) confirmed these risk factors and proved the importance of CL and hand hygiene, avoidance of CLs exposure to contaminated water, use of effective CL disinfection solutions, or use of disposable CLs in reducing the $\mathrm{AK}$ incidence.

Acanthamoeba genus was divided into 20 different genotypes (T1 to T20) based on the variation of ribosomal RNA nucleotide sequences (Fuerst et al, 2015). Each one displayed $5 \%$ or more sequence variations between different genotypes (Corsaro et al, 2015). Genotypes T3, T4, T5, T6, T10, T11, T13, \& T15 cause human Acanthamoeba keratitis (Siddiqui and Khan, 2012).

In the current study, genotype determination was done for nucleotide sequence of positive cases. Partial nucleotide sequences of ASA.S1 region of the $18 \mathrm{~S}$ rDNA gene aligned using ClustalW. The isolated sequ ence in NCBI revealed homology with Acanthamoeba genotype T9 isolate ICS20. The results agreed with Schroeder et al. (2001); Booton et al. (2002) and Kilvington et al. (2004) who found that the strains isolated from lens storage case and soil were both $A$. comandoni of genotype $\mathrm{T} 9$, widely reported as nonpathogenic. In this study, strain was isolated from contact lens storage case of an asymptomatic contact lens wearer without pathogenicity evidence. Orosz et al. (2018) elucidated the identification of Acanthamoe$b a$ isolate belonging to $\mathrm{T} 8$ in corneal sample and fluid from contact lens storage case.

In Egypt, studies reported different environmental prevalent Acanthamoeba genotypes. Lorenzo-Morales et al. (2006) identified 5 genotypes in freshwater sources in the Nile Delta, which were T1, T2, T3, T4 \& T7 genotypes. Hassan et al. (2012) in Alexandria isolated Acanthamoeba from the hydraulic systems of both hemodialysis and den- tal units. Al-Herrawy et al. (2014) identified six Acanthamoeba species from 10 different swimming pools in Cairo; A. polyphaga, A. castellanii, A. rhysodes, A. mauritaniensis, $A$. royreba and $A$. triangularis. Tawfeek et al. (2016) also detected three isolates; T4, T3, \& T5 from environmental sources.

\section{Conclusion}

The culture method proved to be standard test for Acanthamoeba species. It is reliable, cheaper and sensitive than either direct DNA extraction or PCR. There are obstacles with PCR to detect Acanthamoeba especially in corneal swab. The important risk factors that predispose to $\mathrm{AK}$ are related to the contact lens misused. This study confirmed different risk factors association with $\mathrm{AK}$ in CLWs. Genotype determination for Acanthamoeba positive case by PCR revealed homology genotype T9 isolate ICS20. Health education regarding proper CL hygiene and dangers of tap water exposure is important. To our knowledge, this is the first time that Acanthamoeba genotype $\mathrm{T} 9$ is isolated from CLSC in Menoufia Governorate, Egypt.

\section{References}

Aboul-Magd, LA, Abaza, BE, Nada, WM, Mo hammed, FA, Tahaa, AA, et al, 2016: Evaluation of polymerase chain reaction (PCR) as a diagnostic technique for Acanthamoeba keratitis. Parasitol. United J. 9:2:87-94.

Aghajani, A, Dabirzadeh, M, Maroufi, Y, Hooshyar, H, 2016: Identification of Acanthamoe$b a$ genotypes in pools and stagnant water in ponds in Sistan Region in Southeast Iran. Turk. Parazitol. Derg. 40, 3:132-136.

Al-Herrawy, A, Bahgat, M, Mohammed, AE, Ashour, A, Hikal, W, 2014: Acanthamoeba species in swimming pools of Cairo, Egypt. Iran. J. Parasitol. 9, 2:194-201.

Anisah, N, Amal, H, Kamel, AG, Yusof, S, Noraina, AR, Norhayati, M, 2005: Isolation of Acanthamoeba sp. from conjunctival sac of healthy individuals using swab. Trop. Biomed. 22, 1 : 11-14.

Behera, HS, Satpathy, G, 2016: Characterization and expression analysis of trophozoite and cyst proteins of Acanthamoeba spp. isolated from Acanthamoeba keratitis (AK) patient. Mol. Biochem. Parasitol. 205, 1/2:29-34. 
Behera, HS, Satpathy, G, Tripathi, M, 2016: Isolation and genotyping of Acanthamoeba spp. from Acanthamoeba meningitis meningoencephalitis (AME) patients in India. Parasit. Vectors. 9, 1:442-6.

Boggild, AK, Martin, DS, Lee, TY, Yu, B, Low, DE, 2009: Laboratory diagnosis of amoebic keratitis: comparison of four diagnostic methods for different types of clinical specimens. J. Clin. Microbiol. 47, 5:1314-8.

Booton, GC, Kelly, DJ, Chu, YW, Seal, DV, Houang, E, et al, 2002: 18S ribosomal DNA typing and tracking of Acanthamoeba species isolates from corneal scrape specimens, contact lenses, lens cases, and home water supplies of Acanthamoeba keratitis patients in Hong Kong. J. Clin. Microbiol. 40, 5:1621-5.

Booton, GC, Rogerson, A, Bonilla, TD, Seal, DV, Kelly, DJ, et al, 2004: Molecular and physiological evaluation of subtropical environmental isolates of Acanthamoeba spp., causal agent of Acanthamoeba keratitis. J. Eukaryot. Microbiol. 51, 2:192-200.

Buchele, MLC, Wopereis, DB, Casara, F, de Macedo, JP, Rott, MB, et al, 2018: Contact lens-related polymicrobial keratitis: Acanthamoeba spp. genotype T4 and Candida albicans. Parasitol. Res. 117, 11:3431-6.

Carnt, N, Robaei, D, Minassian, DC, Dart, J KG, 2018: Acanthamoeba keratitis in 194 patients: risk factors for bad outcomes and severe inflammatory complications. Br. J. Ophthalmol. 102, 10:1431-5.

Casero, RD, Mongi, F, Laconte, L, Rivero, F, Sastre, D, et al, 2017: Molecular and morphological characterization of Acanthamoeba isolated from corneal scrapes and contact lens wearers in Argentina. Infect. Genet. Evol. 54:70-175.

Cateau, E, Delafont, V, Hechard, Y, Rodier, MH, 2014: Free-living amoebae: What part do they play in healthcare-associated infections? J. Hosp. Infect. 87, 3:131-40.

Corsaro, D, Walochnik, J, Köhsler, M, Rott, MB, 2015: Acanthamoeba misidentification and multiple labels: redefining genotypes T16, T19, \& T20 and proposal for Acanthamoeba micheli sp. nov. (genotype T19). Parasitol. Res. 114: 2481-90.

Dart, JK, Saw, VP, Kilvington, S, 2009: Acanthamoeba Keratitis: diagnosis and treatment update 2009. Am. J. Ophthalmol. 148:487-99.

El-Sayed, NM, Younis, MS, Elhamshary, A M, Abd-Elmaboud, AI, Kishik SM, 2014: Ac- anthamoeba DNA can be directly amplified from corneal scrapings. Parasitol. Res. 113, 9: 3267-72.

El-Sayed, NM Hikal, WM, 2015: Several staining techniques to enhance the visibility of Acanthamoeba cysts. Parasitol. Res. 114, 3:823-30.

Evyapan, G, Koltas, IS, Eroglu, F, 2015: Genotyping of Acanthamoeba T15: The environmental strain in Turkey. Trans. R. Soc. Trop. Med. Hyg. 109, 3:221-4.

Fuerst, PA, Booton, GC, Crary, M, 2015:

Phylogenetic analysis and the evolution of the 18S rRNA gene typing system of Acanthamoeba. J. Eukaryot. Microbiol. 62, 1:69-84.

Garcia, LS, Bruckner, DA, 1997: Macroscopic and microscopic examination of fecal specimens. In: Diagnostic Medical Parasitology $\left(3^{\text {rd }}\right.$ Ed.). ASM Press; Washington, DC.

Gatti, S, Rama, P, Matuska, S, Berrilli, F, Cavallero, $\mathrm{A}$, et al, 2010: Isolation and genotyping of Acanthamoeba strains from corneal infections in Italy. J. Med. Microbiol. 59, 11:1324-30.

Ghamilouie, MM, Valadkhani, Z, Rahimi, F, Khoshzaban, F, Aghighi, Z, et al, 2014: Isolation \& genotyping of Acanthamoeba strains from corneal scraps. Iran. J. Ophthalmol. 26, 2:97100.

Goldschmidt, P, Degorge, S, Saint-Jean, C, Yera, H, Zekhnini, F, et al, 2008: Resistance of Acanthamoeba to classic DNA extraction methods used for the diagnosis of corneal infections. Br. J. Ophthalmol. 92, 1:112-5.

Gomes, T dos S, Magnet, A, Izquierdo, F, Vaccaro, L, Redondo, F, et al, 2016: Acanthamoeba spp. in contact lenses from healthy individuals from Madrid, Spain. PLoS One 11, 4: e0154246.

Gupta, S, Aher, A, 2009: Acanthamoeba keratitis: A case report. People J. Sci. Res. 2, 2:9-11.

Hassan, A, Farouk, H, Hassanein, F, AbdulGhani, R, Abdelhady, AH, 2012: Acanthamoe$b a$ contamination of hemodialysis and dental units in Alexandria, Egypt: a neglected potential infection source. J. Infect. Publ. Hlth. 5:304-10.

Ibrahim, YW, Boase, DL, Cree, IA, 2009: How could contact lens wearers be at risk of Acanthamoeba infection? a review. J. Optom. 2, 2: 60-6.

Ithoi, I, Ahmad, AF, Mak, JW, Nissapatorn, V, Lau, YL, et al, 2011: Morphological characteristics of developmental stages of Acantham$o e b a$ and Naegleria species before and after staining by various techniques. Southeast Asian J. 
Trop. Med. Publ. Hlth. 42, 6:1327-38.

Khan, NA, 2006: Acanthamoeba biology and increasing importance in human health. FEMS Microbiol. Rev. 30:564-95.

Kilvington, S, Gray, T, Dart, J, Morlet, N, Beeching, JR, et al, 2004: Acanthamoeba keratitis: The role of domestic tap water contamination in the United Kingdom. Invest. Ophthalmol. Vis. Sci. 45, 1: 165-9.

Laummaunwai, P, Ruangjirachuporn, W, Boonmars, T, 2012: A simple PCR condition for detection of a single cyst of Acanthamoeba species. Parasitol. Res. 110, 4:1569-72.

Legarreta, JE, Nau, AC, Dhaliwal, DK, 2013: Acanthamoeba keratitis associated with tap water use during contact lens cleaning: manufacturer guidelines need to change. Eye Contact Lens. 39, 2:158-61.

Liesegang, TJ, 2002: Physiologic changes of the cornea with contact lens wear. CLAO J. 28, $1: 12-27$

Lorenzo-Morales, J, Ortega-Rivas, A, Martínez, E, Khoubbane, M, Artigas, P, et al, 2006: Acanthamoeba isolates belonging to T1, T2, T3, $\mathrm{T} 4$ and $\mathrm{T} 7$ genotypes from environmental freshwater samples in the Nile Delta region, Egypt. Acta Trop. 100, 1/2:63-9.

Lorenzo-Morales, J, Morcillo-Laiz, R, Martín-Navarro, CM, López-Vélez, R, López-Arencibia, A, et al, 2011: Acanthamoeba keratitis due to genotype T11 in a rigid gas permeable contact lens wearer in Spain. Cont. Lens Anterior Eye 34, 2:83-6.

Lorenzo-Morales, J, Khan, NA, Walochnik, J, 2015: An update on Acanthamoeba keratitis: diagnosis, pathogenesis and treatment. Parasite 22:10-2.

Maciver, SK, Asif, M, Simmen, MW, Lorenzo -Morales, J, 2013: A systematic analysis of Acanthamoeba genotype frequency correlated with source and pathogenicity: T4 is confirmed as a pathogen-rich genotype. Eur. J. Protistol. 49, 2: 217-21.

Mahittikorn, A, Kittichathanakul, T, To-Im, J, Nacapunchai, D, 2017: Knowledge, behavior, and free-living amoebae contamination of cosmetic contact lens among University wearers in Thailand: A cross-sectional study. Eye Cont. Lens 43, 2:81-8.

Niyyati, M, Lorenzo-Morales, J, Rezaie, S, Rahimi, F, Mohebali, M, et al, 2009: Genotyping of Acanthamoeba isolates from clinical and environmental specimens in Iran. Exp. Parasitol.
$121,3: 242-5$

Omaña-Molina, M, Vanzzini-Zago, V, Hernandez-Martinez, D, Gonzalez-Robles, A, Salazar-Villatoro, L, et al, 2016: Acanthamoeba genotypes T3 and T4 as causative agents of amoebic keratitis in Mexico. Parasitol. Res. 115, 2: 873-8.

Orosz, E, Szentmáry, N, Kiss, HJ, Farkas, A, Kucsera, I, et al, 2018: First report of Acanthamoeba genotype T8 human keratitis. Acta Microbiol. Immunol. Hung. 65, 1:73-9.

Page, FC, 1988: A new key to fresh water and soil amoebae. In: Freshwater Biological Association Scientific Publications, Cambria, UK.

Pussard, M, Pons, R, 1977: Morphologies de la paroi kystique et taxonomie du genre Acanthamoeba (Protozoa, Amoebida). Protistol. 13:557610.

Qvarnstrom, Y, Visvesvara, GS, Sriram, R, da Silva, AJ, 2006: Multiplex real-time PCR assay for simultaneous detection of Acanthamoeba spp., Balamuthia mandrillaris, and Naegleria fowleri. J. Clin. Microbiol. 44, 10:3589-95.

Rezaeian, M, Niyyati, M, Farnia, S, Motevalli-Haghi, A, 2008: Isolation of Acanthamoeba Spp. from different environmental sources. Iran. J. Parasitol. 3:44-7.

Schroeder, JM, Booton, GC, Hay, J, Niszl, I A, Seal, DV, et al, 2001: Use of subgenic 18S ribosomal DNA PCR and sequencing for genus and genotype identification of Acanthamoebae from humans with keratitis and from sewage sludge. J. Clin. Microbiol. 39:1903-11.

Schuster, FL, 2002: Cultivation of pathogenic and opportunistic free-living amebas. Clin. Microbiol. Rev. 15, 3: 342-54.

Siddiqui, R, Khan, NA, 2012: Biology and pathogenesis of Acanthamoeba. Parasit. Vectors 5: 6-9.

Srinivasan, S, Otchere, H, Yu, M, Yang, J, Luensmann, D, Jones, L, 2015: Impact of cosmetics on the surface properties of silicone hydrogel contact lenses. Eye Cont. Lens 41, 4:22835.

Taher, EE, Méabed, EMH, Abdallah, I, Abdel Wahed, WY, 2018: Acanthamoeba keratitis in noncompliant soft contact lenses users: Genotyping and risk factors, a study from Cairo, Egypt. J. Infect. Publ. Hlth. 11, 3:377-83.

Tamura, K, Nei, M, Kumar, S, 2004: Prospects for inferring very large phylogenies by using the neighbor-joining method. Proc. Natl. Acad. Sci. USA. 101, 30:11030-5. 
Tamura, K, Stecher, G, Peterson, D, Filipski, A, Kumar, S, 2013: MEGA6: Molecular evolutionary genetics analysis version 6.0. Mol. Biol. Evol. 30, 12:2725-9.

Tawfeek, GM, Bishara, SA, Sarhan, RM, Taher, EE, Khayyal, AE, 2016: Genotypic, physiological, and biochemical characterization of potentially pathogenic Acanthamoeba isolated from the environment in Cairo, Egypt. Parasitol. Res. 115, 5:1871-81.

Vemuganti, GK, Sharma, S, Athmanathan, S, Garg, P, 2000: Keratocyte loss in Acanthamoe- ba keratitis: phagocytosis, necrosis or apoptosis. Indian J. Ophthalmol. 48, 4:291-4.

Visvesvara, GS, 2013: Infections with free-living amebae. Handb. Clin. Neurol. 114:153-68.

Walochnik, J, Scheikl, U, Haller-Schober, E M, 2015: Twenty years of Acanthamoeba diagnostics in Austria. J. Eukaryot. Microbiol. 62, 1:3-11.

Wanachiwanawin, D, Booranapong, W, Kosrirukvongs, P, 2012: Clinical features of Acanthamoeba keratitis in contact lens wearers and non-wearers. Southeast Asian J. Trop. Med. Publ. Hlth. 43, 3: 549-56.

\section{Explanation of figures}

Fig. 1: Distribution of Acanthamoeba infection in different groups examined by different techniques

Fig. 2: Direct wet mount mlsmear (x1000), Scale bar 20um. a: Acanthamoeba trophozoite $(\mathrm{T}), \mathrm{N}=$ nucleus, Ps= pseudopodium. b- Cysts of subgroup II, $\mathrm{A}=$ ectocyst, $\mathrm{B}=$ endocyst, $\mathrm{N}=$ =nucleus. $\mathrm{C}$ - Cyst of subgroup I (red arrow)

Fig. 3: Acanthamoeba trophozoite (a) and cysts (b) with Giemsa stain. Acanthamoeba trophozoite (c) and cysts (d) with trichrome stain (x1000). $\mathrm{T}=$ trophozoite, $\mathrm{N}=$ nucleus, $\mathrm{a}=$ acanthopodia. Scale bar $=20 \mu \mathrm{m}$.

Fig. 4: Agarose gel electrophoresis of PCR products, Lane 1=DNA ladder (100-1000 bp), Lane 2= +ve control, Lane 3= -ve control, Lane 4= -ve sample, Lane 5= +ve sample, and Lanes 6,7 and $8=-$ ve samples.

Fig. 5: Phylogenetic tree including strain NA-2015. Evolutionary distances were computed using Maximum Composite Likelihood method. Phylogenetic analysis conducted in MEGA6 software program using Neighbor-Joining method.
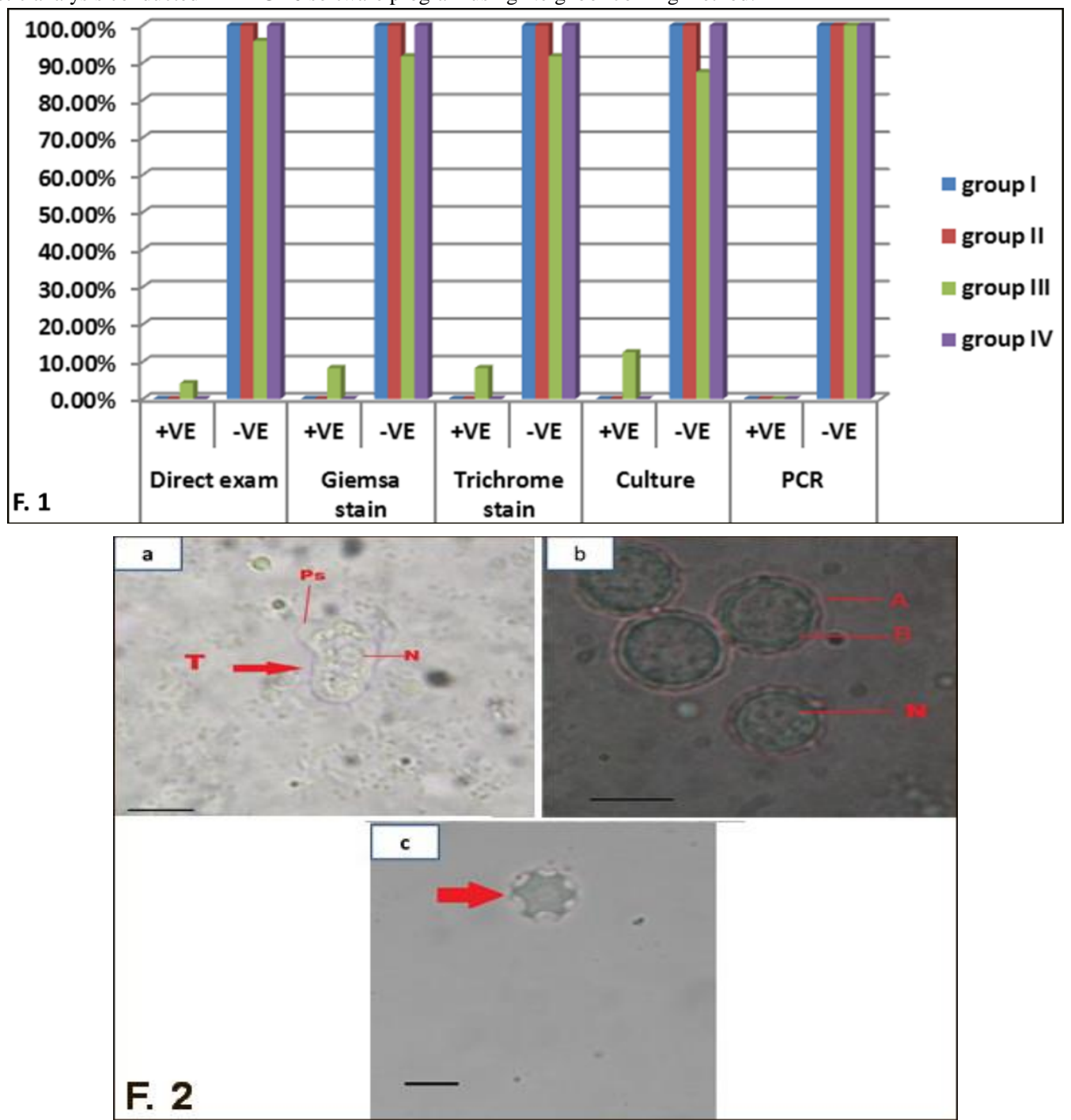

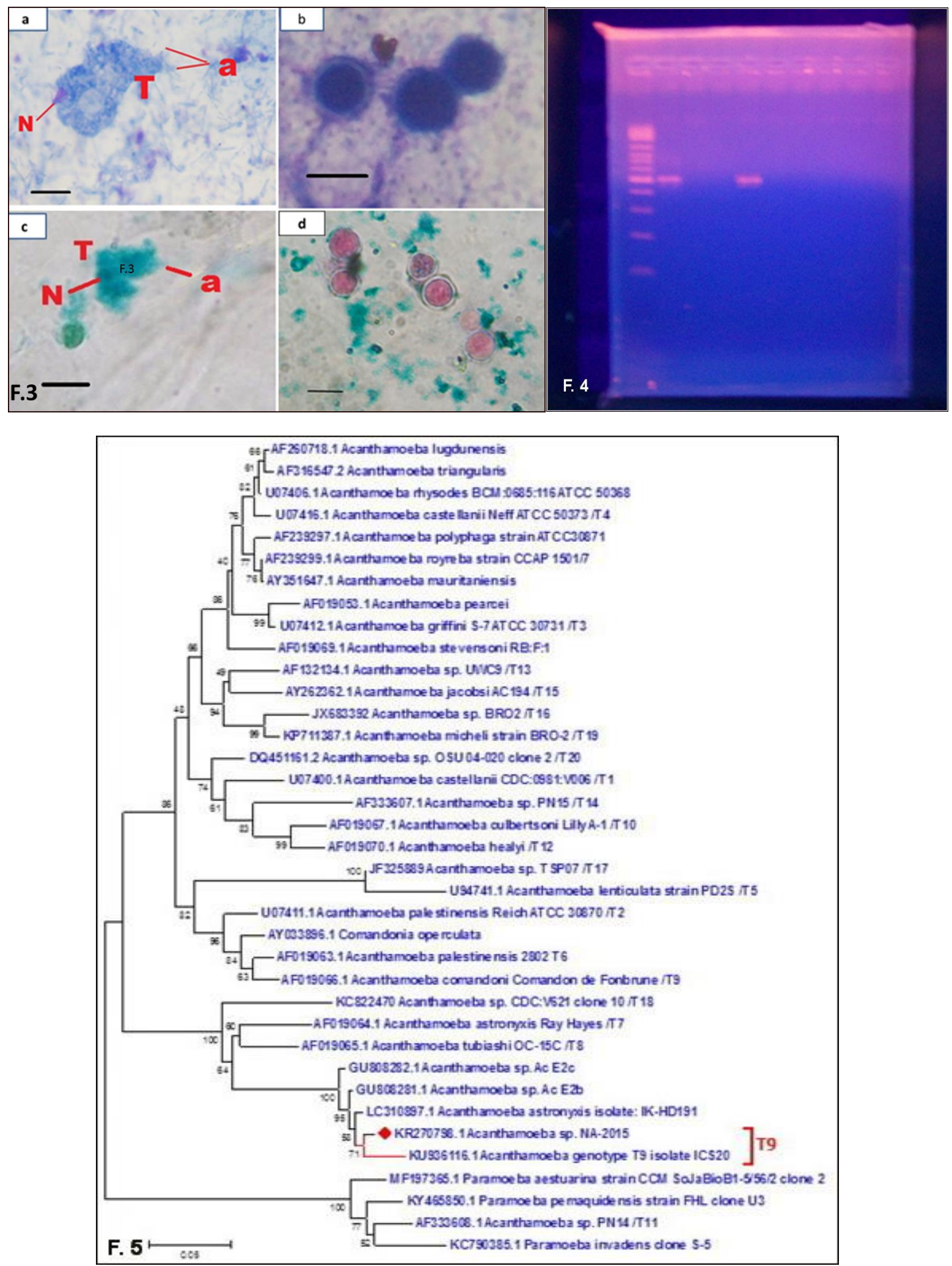